\title{
Picking up the pace: Effects of global temporal context on sensitivity to the tempo of auditory sequences
}

\author{
J. Devin McAuley and Nathaniel S. Miller \\ Bowling Green State University, Bowling Green, Ohio
}

\begin{abstract}
This article continues a line of research examining factors affecting listeners' auditory tempo sensitivities. Of particular interest is the question of whether listeners are sensitive to the overall (global) pace of their auditory environment and how this sensitivity may affect their perceptions of sequence timing. To address this question, we manipulated the set of sequence tempi (between 300 and $700 \mathrm{msec}$ ) that listeners experienced over the course of a 1-h period (i.e., the global temporal context) while they performed a tempo-discrimination task involving standard-comparison pairs of isochronous tone sequences. Overall findings show systematic distortions in perceived tempo that are consistent with the view that listeners adapt to the global pace of their auditory environments. Moreover, general support was found for the hypothesis that increasing the number of equal intervals in a standard sequence produces greater improvements in tempo sensitivity when the standard sequence tempo is different from the global pace than when it is at the global pace. Implications of these findings for models of timing and temporal processing are discussed.
\end{abstract}

A fundamental question that has challenged psychologists concerns the temporal coordination of behavior with the dynamic patterning of events (Fraisse, 1963; Jones, 1976; Lashley, 1951). In the world, individuals have the potential to be sensitive to both local and global aspects of environmental timing. On a local level, rhythmic regularity of short sequences of events conveys to individuals a sense of sequence tempo, affording predictions about when the next event in a sequence is likely to occur. On a more global level, average event rate-determined over an extended time period-conveys to individuals information about the overall (global) pace of environmental events. This article continues a line of research concerned with the nature of the mechanism(s) underpinning human timing and the role that local and global temporal context play in time judgment behavior (Jones \& McAuley, 2005; McAuley \& Jones, 2003; McAuley \& Kidd, 1998; Miller $\&$ McAuley, 2005). Of particular interest in the present research is the issue of global pace in the auditory domain and how it may affect the listener's sensitivity to local sequence tempo.

Studies examining human tempo sensitivity have generally found that judgments about the relative tempo of tone sequences (i.e., whether a sequence is "faster" or "slower" than a preceding sequence) are more accurate for sequences that have regular (rhythmic) timing than irregular timing (Drake \& Botte, 1993; Jones \& Yee, 1997). One commonly used task to assess questions about sensitivity to sequence tempo is illustrated in Figure 1. For this task, participants are presented with standard-comparison pairs of isochronous tone sequences and are asked to judge the tempo of the comparison with the standard, responding "faster" or "slower." Assessments of tempo sensitivity using isochronous sequences generally show that as the number of sequence intervals increases, participants' tempo sensitivities improve (Drake \& Botte, 1993; Grondin, 2001; McAuley \& Kidd, 1998). For a limited range of time intervals, relative just-noticeable differences (JNDs) for both tempo and duration typically average $6 \%$ for single-interval (two-tone) sequences (Abel, 1972; Allan, 1979; Creelman, 1962; Getty, 1975; Miller \& McAuley, 2005; Small \& Campbell, 1962; Woodrow, 1951), whereas JNDs for multiple-interval sequences are sometimes less than about 2\% (Drake \& Botte, 1993; Friberg \& Sundberg, 1995; Michon, 1964).

To explain improvements associated with the number of sequence intervals, Drake and Botte (1993) proposed a multiple-look model whereby each interonset interval (IOI) in the standard sequence provides an independent but variable estimate of sequence tempo. They hypothesized (as have others) that listening to the standard sequence leads to separate interval-based estimates of the standard's tempo, which are averaged to form an aggregate memory trace (Drake \& Botte, 1993; Ivry \& Hazeltine, 1995; Keele, Nicoletti, Ivry, \& Pokorny, 1989; Schulze, 1989). As the number of independent "looks" at the same standard IOI increases, the average sampling error between the estimated and actual tempo decreases, leading to lower

J. D. McAuley, mcauley@bgnet.bgsu.edu 


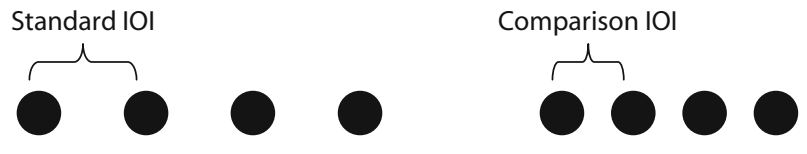

Standard-Comparison Sequence Pair

Figure 1. Diagram illustrating a typical tempo-discrimination task. Participants hear standard-comparison pairs of isochronous tone sequences and judge the tempo of the second (comparison) sequence in comparison with the first (standard) sequence. The tempo of each sequence was defined by the fixed interonset interval (IOI) between tone onsets, labeled in the diagram as Standard IOI and Comparison IOI, respectively.

discrimination thresholds. Drake and Botte predicted that the JND in tempo - taken as the standard deviation of the sampling distribution-should decrease in inverse proportion to the square root of the number of sequence intervals, as shown below.

$$
J N D_{n}=\frac{J N D_{1}}{\sqrt{n}} .
$$

In this equation, $J N D_{1}$ is the observed JND for a singleinterval standard sequence and $J N D_{n}$ is the predicted JND for an $n$-interval standard sequence.

Consistent with the multiple-look model, multipleinterval advantages have been reported for both auditory and visual sequences for tasks involving time-interval perception as well as production (Grondin, 2001; Ivry \& Hazeltine, 1995; McAuley \& Jones, 2003; McAuley \& Kidd, 1998; Rousseau \& Rousseau, 1996; ten Hoopen \& Akerboom, 1983). One problem in the interpretation of some of this work, however, is that the number of intervals in the standard and comparison sequences frequently covary, making the precise reason for the observed improvements unclear (Drake \& Botte, 1993; Grondin, 2001; McAuley $\&$ Kidd, 1998). That is, although Drake and Botte and others have assumed that the improvements are due to the number of standard intervals, it is unclear in many cases whether the multiple-interval advantage is indeed due to the number of standard intervals or the number of comparison intervals, or both.

Miller and McAuley (2005) distinguished these three possibilities using a design similar to that of Drake and Botte (1993). In two experiments, they independently varied the number of standard and comparison intervals in standard-comparison pairs of isochronous tone sequences and found - somewhat surprisingly - that with a fixed 500 -msec standard IOI on each trial, the number of standard intervals had little to no effect on tempo sensitivity; rather, improvements in tempo sensitivity appeared to be due solely to the number of intervals in the comparison sequence. With a roving standard IOI, however, a different picture emerged. When the standard IOI varied from trial to trial over the course of the experiment, tempo sensitivity was affected by the number of both standard and comparison intervals. Closer inspection of the data suggested that the number of standard intervals appeared to have an effect on thresholds for only the two standard IOIs (400 and $600 \mathrm{msec}$ ) that differed from the mean 500-msec stan- dard IOI that listeners experienced in the experiment (i.e., $500 \mathrm{msec}$ ). These findings suggest that listeners might be developing a stable tempo referent for the average sequence rate that is experienced in the experiment (i.e., the global pace). Support for this interpretation was found in a comparison of estimates of points of subjective equality (PSE) for the different standard IOIs. This comparison showed a systematic pattern of over- and underestimation for standard IOIs that were shorter and longer than the mean standard IOI, respectively, reminiscent of Vierordt's (1868) law. Thus, in the context of a tempo-discrimination task, judgments about standard sequence tempi that differ from the global pace appeared to be pulled in the direction of the global pace, and this result has the potential to influence the relative contribution of the number of standard and comparison intervals to tempo thresholds.

The present study extends that of Miller and McAuley (2005) in several respects. First, it more directly examines the question of sensitivity to global pace by manipulating the mean standard IOI (thus, the global pace) that different groups of listeners experience while they are performing a tempo-discrimination task. Second, it provides a test of a hypothesis that increasing the number of standard intervals provides greater facilitation of tempo judgments when the standard tempo is different from the global pace than when it is at the global pace. Finally, it rules out the possibility that the results reported earlier may have been somehow specific to testing a 500-msec standard IOI. In Miller and McAuley, the mean standard IOI in the roving standard IOI condition was equal to $500 \mathrm{msec}$, which was also the same value that was used in the fixed standard IOI condition. Thus, it is possible that the null effect of the number of standard intervals observed for this IOI in the roving standard condition may have had nothing to do with participants developing a stable referent for the mean sequence rate; rather, it may have been due instead to the specific absolute value of the standard that was chosen.

To conclusively rule out this possibility, we controlled for the absolute value of the standard IOI by placing a 500 -msec standard IOI in one of two global contexts, which we will refer to as the 345 context and the 567 context. In the 345 context, standard IOIs varied between 300,400 , and $500 \mathrm{msec}$; in this case, a 500-msec standard IOI results in a relatively slow tempo in comparison with the mean standard IOI of the global context. Conversely, in the 567 context, standard IOIs varied between 500, 600, and $700 \mathrm{msec}$; in this case, a $500-\mathrm{msec}$ standard IOI results in a relatively fast tempo in comparison with the mean standard IOI of the global context.

If the previous results generalize, then we would expect to replicate the results of McAuley and Miller (2005; Experiment 2) for the 345 and 567 contexts. Moreover, as indicated previously, the design permits the additional comparison of the same standard IOI $(500 \mathrm{msec})$ in different relative temporal positions within each global temporal context. In the 567 context, the 500-msec standard IOI specifies a standard sequence with a relatively fast tempo compared with the global pace and, in the 345 context, the 500-msec standard IOI specifies a standard sequence 
with a relatively slow tempo compared with the global pace. This design can be contrasted with that of Miller and McAuley (2005; Experiment 2), in which the 500-msec standard IOI specified a standard sequence that was at the global pace. Overall, if participants are sensitive to global pace, we would expect PSEs and associated constant error scores (CEs) for each standard IOI to vary systematically as a function of the standard sequences' relative temporal positions within the global temporal context. Moreover, we would expect to find larger effects of the number of standard intervals on tempo sensitivity when standard IOIs are different from the global pace (i.e., the standard IOI specifies a sequence tempo that is relatively fast or slow in comparison with the global pace) than when the standard IOI specifies a sequence tempo that is at the global pace.

\section{METHOD}

\section{Design}

The experiment implemented a 2 (number of standard intervals) $\times 2$ (number of comparison intervals) $\times 2$ (context) $\times 3$ (standard IOI) mixed factorial design. Standard sequences with $\left(n_{1}=1,3\right)$ intervals were crossed with comparisons sequences with $\left(n_{2}=1,3\right)$ intervals, yielding four sequence conditions that varied between subjects; see Figure 2. To examine the effects of global temporal context, we manipulated the set of standard IOIs that participants experienced during the experiment. In a 345 context, the standard IOI was either 300,400 , or $500 \mathrm{msec}$; in a 567 context, the standard IOI was either 500,600 , or $700 \mathrm{msec}$. For both contexts, the standard IOI varied randomly from trial to trial and took on one of three values.

\section{Participants}

A total of 102 undergraduate students at Bowling Green State University with self-reported normal hearing participated in the experiment in return for extra credit in an introductory psychology course. Participants were randomly assigned to one of the four standard-comparison sequence conditions and placed in one of the two global contexts. Across all conditions, the data from ten participants were discarded because of inattention, failure to follow task instructions, or because preliminary threshold estimates were excessively large $(>20 \%)$. For the 345 and 567 contexts, final participant numbers for the $1: 1,1: 3,3: 1$, and $3: 3$ sequence conditions were $(n=$ $12,12,12$, and 13$)$ and $(n=10,11,9$, and 13$)$, respectively.

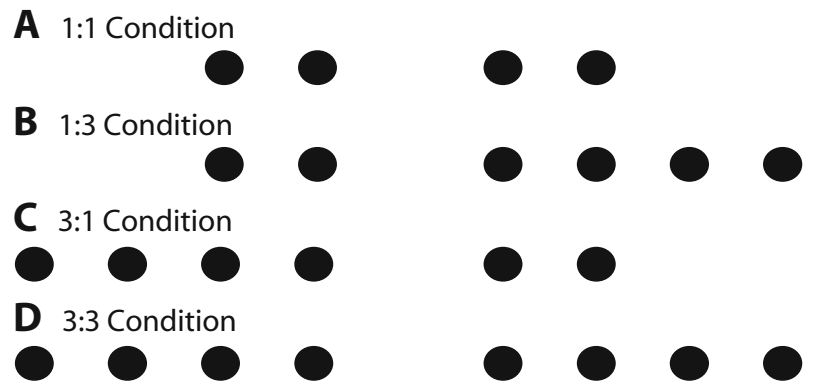

Figure 2. Different sequence conditions are labeled $\left(n_{1}: n_{2}\right)$ to specify the number of equal intervals in the standard sequence $\left(n_{1}\right)$ and the number of equal intervals in the comparison sequence $\left(n_{2}\right)$. Consistent with previous research, the gap between the standard and comparison sequences was defined as the time interval between the onset of the late tone of the standard sequence and the first tone of the comparison sequence and was always equal to twice the duration of the standard interonset interval.

\section{Stimuli and Equipment}

Tone sequences were presented to participants at a comfortable listening level through Koss TI/65 headphones that were attached to a Yamaha PSR-270 MIDI keyboard that was set to a grand piano voice. All tones were $50 \mathrm{msec}$ in duration, with a $440-\mathrm{Hz}$ fundamental. For each standard-comparison sequence pair, the time interval between the onset of the last tone of the standard sequence and the first tone of the comparison sequence was equal to twice the standard IOI. Comparison IOIs varied randomly from trial to trial and took on values that were yoked to the standard IOI. Stimulus generation and response collection were controlled by the MIDILAB software with a time resolution of $\approx 1 \mathrm{msec}$ (Todd, Boltz, \& Jones, 1989).

\section{Procedure}

Participants listened to each standard-comparison pair of sequences and judged whether the comparison sequence was faster or slower than the standard. Prior to testing, participants received instructions about the tempo judgment task while they studied a diagram of the randomly assigned sequence condition $(1: 1,1: 3$, $3: 1$, or $3: 3$ ). They were then given a practice block of 24 trials with feedback that included examples of each tested standard IOI (345 context, 300, 400, $500 \mathrm{msec} ; 567$ context, 500, 600, $700 \mathrm{msec}$ ). For the practice block, tempo differences were $\pm 15 \%$ and $\pm 30 \%$. Following practice, participants were administered five test blocks of 36 trials with no feedback. For test blocks, standard and comparison IOIs varied randomly from trial to trial; comparison IOIs were either $\pm 2 \%, \pm 6 \%$, or $\pm 10 \%$ in comparison with the standard IOI. Over the course of the test blocks, ten observations were obtained for each standard-comparison pair. The order of trials within a block was randomized and the order of test blocks was counterbalanced across participants. In total, the experiment lasted approximately $90 \mathrm{~min}$, with short breaks between test blocks.

\section{Data Analysis}

Proportions of "faster" responses were determined for each of the six comparison IOI values and averaged over the five test blocks. JNDs and PSEs were then determined from the resulting cumulative response curves by using the method that was described by Macmillan and Creelman (1991, pp. 219-220). In this method, the JND is measured by half the distance between the 25 th and 75 th percentiles, which we then converted to a Weber fraction by dividing the JND by the standard IOI. Similarly, the PSE is measured by the point on the cumulative response curve that corresponds to a comparison IOI judged "faster" $50 \%$ of the time, which-for comparison with previous work - we converted to a relative constant error (CE) score: $\mathrm{CE}=(\mathrm{PSE}-$ standard IOI $) /$ standard IOI. Positive values for CE imply overestimation of the standard IOI, whereas negative values imply underestimation of the standard IOI. $R^{2}$ measures of goodness-of-fit for the estimated psychometric curves ranged between .83 and .95 across conditions.

In order to compare the two context conditions in the same analysis, standard IOI was coded in comparison with the mean standard IOI (global pace) of the session ( $400 \mathrm{msec}$ for the 345 context; $600 \mathrm{msec}$ for the 567 context), yielding matching values of -100 , 0 , and $100 \mathrm{msec}$ for the three standard IOIs in each context. Separate $2 \times 2 \times 2 \times 3$ mixed measures ANOVAs were performed on relative JNDs and CEs to assess the effects of the number of standard intervals ( 1 vs. 3 ), number of comparison intervals ( 1 vs. 3 ), context ( 345 vs. 567$)$, and standard IOI $(-100,0,100)$; standard IOI was the only factor with repeated measures.

\section{RESULTS}

The results are presented in three sections. First, we describe the overall pattern of results for the JND and CE for the 345 and 567 context conditions. Second, we control for potential differences associated with the absolute magnitude of the standard IOI by comparing the JND and 
CE results for just the 500-msec standard IOI in the 345 and 567 contexts. Finally, we consider the emergence of sensitivity to "global pace" by examining changes in CE for the 500-msec standard IOI across test blocks.

\section{Overall Pattern of JNDs and CEs in 345 and 567 Contexts}

JND results by sequence condition for the 345 (fast pace) and 567 (slow pace) contexts are shown in Table 1. For both contexts, thresholds were highest in the 1:1 condition, next highest in the 1:3 and 3:1 conditions, and lowest in the 3:3 condition. Consistent with independent contributions of the number of standard and comparison intervals to tempo thresholds, the ANOVA on relative JNDs revealed main effects of number of standard intervals $\left[F(1,85)=14.75, M S_{\mathrm{e}}=13.86, p<.01, \eta_{\mathrm{p}}^{2}=\right.$ $0.15]$, and number of comparison intervals $[F(1,85)=$ $\left.18.8, M S_{\mathrm{e}}=13.86, p<.01, \eta_{\mathrm{p}}^{2}=0.18\right]$, but no interaction between these factors $\left[F(1,85)=1.93, M S_{\mathrm{e}}=13.86\right.$, $\left.p=.17, \eta_{\mathrm{p}}^{2}=0.02\right]$. Overall, there was no main effect of context, but there was a marginal interaction between context and the number of standard intervals $[F(1,85)=$ $\left.3.85, M S_{\mathrm{e}}=13.86, p=.053, \eta_{\mathrm{p}}^{2}=0.04\right]$; Table 1 suggests that the effect of the number of standard intervals was slightly stronger in the 567 context than in the 345 context. Although there was no main effect of the standard IOI or significant interactions (all $p \mathrm{~s}>.2$ ), separate ANOVAs for each of the three standard IOIs indicated that the magnitude of the effect of the number of standard intervals was much larger when the standard IOI was different from the global pace $\left(\eta_{\mathrm{p}}^{2}=0.17\right)$ than when it was at the global pace $\left(\eta_{\mathrm{p}}^{2}=0.04\right)$.

Table 2 illustrates the general pattern of results for the $\mathrm{CE}$ measure. In this table, the average $\mathrm{CE}$ is plotted for each value of the standard IOI in the 345 and 567 contexts. The most striking aspect of the $\mathrm{CE}$ results was that errors were sensitive to the average (global) pace of events; independent of the absolute value of the standard IOI, sequence rates faster and slower than the global pace were overestimated and underestimated, respectively, whereas the rate (i.e., standard IOI) equal to the global pace produced the least amount of error. This finding was confirmed by the ANOVA on CE, which revealed a significant main effect of standard IOI $\left[F(2,170)=83.3, M S_{\mathrm{e}}=28.03, p<.01\right.$, $\left.\eta_{\mathrm{p}}^{2}=0.50\right]$.
The ANOVA on CE also revealed several significant interactions. Specifically, we observed a two-way interaction between standard IOI and the number of standard intervals $\left[F(2,170)=11.15, M S_{\mathrm{e}}=28.03, p<.01\right.$, $\left.\eta_{\mathrm{p}}^{2}=0.12\right]$, as well as three-way interactions between standard IOI, context, and number of standard intervals $\left[F(2,180)=4.05, M S_{\mathrm{e}}=28.03, p<.05, \eta_{\mathrm{p}}^{2}=0.05\right]$, between standard IOI, context, and number of comparison intervals $\left[F(2,170)=4.37, M S_{\mathrm{e}}=28.03, p<.05, \eta_{\mathrm{p}}^{2}=\right.$ $0.05]$, and between standard IOI and numbers of standard and comparison intervals $\left[F(2,170)=5.88, M S_{\mathrm{e}}=28.03\right.$, $\left.p<.01, \eta_{\mathrm{p}}^{2}=0.07\right]$.

A close inspection of Table 2 permits several generalizations that provide some insight about the observed higher order interactions. First, the degree of over- and underestimation of fast and slow sequence rates was generally larger in the 345 context than in the 567 context (see column means in Table 2). Second, increasing the number of standard intervals generally reduced the magnitude of errors $\left(n_{1}=1, M=1.36 ; n_{1}=3, M=0.10\right)$. Third, the $1: 3$ sequences were particularly disruptive, with the magnitude of errors generally increasing in a manner that was sensitive to sequence rate in comparison with global pace.

\section{Comparison of 500-msec Standard IOI Across Contexts}

Next, we considered performance for only the 500 -msec standard IOI, which appeared in both the 345 (fast pace) and 567 (slow pace) contexts. This analysis permitted us to control for potential differences associated with the absolute magnitude of the standard IOI. For this analysis, ANOVAs on relative JNDs and CEs were conducted for just the 500-msec standard IOI with context, number of standard intervals, and number of comparison intervals as between-subjects factors.

For the ANOVA on relative JNDs, there was a main effect of the number of standard intervals $[F(1,84)=15.52$, $\left.M S_{\mathrm{e}}=5.92, p<.01, \eta_{\mathrm{p}}^{2}=0.16\right]$, a main effect of the number of comparison intervals $\left[F(1,84)=12.11, M S_{\mathrm{e}}=5.92\right.$, $\left.p<.01, \eta_{\mathrm{p}}^{2}=0.07\right]$, but no interaction between these factors $\left[F(1,84)=0.57, M S_{\mathrm{e}}=5.92, p=.45, \eta_{\mathrm{p}}^{2}=0.007\right]$. For the ANOVA on CE, there was a main effect of context $\left[F(1,84)=61.7, M S_{\mathrm{e}}=35.18, p<.01, \eta_{\mathrm{p}}^{2}=0.42\right]$. Average relative $\mathrm{CE}$ scores for the $500-\mathrm{msec}$ standard IOI in

Table 1

Relative Just-Noticeable Difference (JND) Reported As a Percentage of the Standard Interonset Interval (IOI) and Standard Error of the Mean of the Four Sequence Conditions (1:1, 1:3, 3:1, and 3:3) for the Three Standard IOIs in the 345 (Fast Pace) and 567 (Slow Pace) Contexts

\begin{tabular}{|c|c|c|c|c|c|c|c|c|c|c|c|c|}
\hline \multirow{3}{*}{$\begin{array}{l}\text { Sequence } \\
\text { Condition }\end{array}$} & \multicolumn{6}{|c|}{345 Context } & \multicolumn{6}{|c|}{567 Context } \\
\hline & \multicolumn{2}{|c|}{$300 \mathrm{msec}$} & \multicolumn{2}{|c|}{$400 \mathrm{msec}$} & \multicolumn{2}{|c|}{$500 \mathrm{msec}$} & \multicolumn{2}{|c|}{$500 \mathrm{msec}$} & \multicolumn{2}{|c|}{$600 \mathrm{msec}$} & \multicolumn{2}{|c|}{$700 \mathrm{msec}$} \\
\hline & JND & $S E$ & JND & $S E$ & JND & $S E$ & JND & $S E$ & JND & $S E$ & JND & $S E$ \\
\hline $1: 1$ & 7.34 & 0.75 & 7.52 & 0.84 & 7.56 & 0.76 & 6.59 & 0.69 & 7.83 & 0.96 & 8.67 & 0.68 \\
\hline $1: 3$ & 5.94 & 0.75 & 6.90 & 0.84 & 6.04 & 0.76 & 6.31 & 0.66 & 5.68 & 0.92 & 6.67 & 0.65 \\
\hline $3: 1$ & 6.94 & 0.89 & 7.78 & 1.08 & 6.05 & 0.76 & 4.86 & 0.73 & 6.53 & 1.02 & 5.09 & 0.72 \\
\hline $3: 3$ & 4.71 & 0.72 & 4.74 & 0.80 & 4.37 & 0.73 & 3.18 & 0.60 & 3.06 & 0.84 & 3.39 & 0.60 \\
\hline$M$ & 6.23 & 0.38 & 6.51 & 0.42 & 6.04 & 0.38 & 5.23 & 0.34 & 5.77 & 0.47 & 5.96 & 0.33 \\
\hline
\end{tabular}


Table 2

Relative Constant Error (CE) Reported As a Percentage of the Standard Interonset Interval (IOI) and Standard Error of the Mean of the Four Sequence Conditions (1:1, 1:3, 3:1, and 3:3) for the Three Standard IOIs in the 345 (Fast Pace) and 567 (Slow Pace) Contexts

\begin{tabular}{|c|c|c|c|c|c|c|c|c|c|c|c|c|}
\hline \multirow{3}{*}{$\begin{array}{l}\text { Sequence } \\
\text { Condition }\end{array}$} & \multicolumn{6}{|c|}{345 Context } & \multicolumn{6}{|c|}{567 Context } \\
\hline & \multicolumn{2}{|c|}{$300 \mathrm{msec}$} & \multicolumn{2}{|c|}{$400 \mathrm{msec}$} & \multicolumn{2}{|c|}{$500 \mathrm{msec}$} & \multicolumn{2}{|c|}{$500 \mathrm{msec}$} & \multicolumn{2}{|c|}{$600 \mathrm{msec}$} & \multicolumn{2}{|c|}{$700 \mathrm{msec}$} \\
\hline & $\mathrm{CE}$ & $S E$ & $\mathrm{CE}$ & $S E$ & $\mathrm{CE}$ & $S E$ & $\mathrm{CE}$ & $S E$ & $\mathrm{CE}$ & $S E$ & $\mathrm{CE}$ & $S E$ \\
\hline $1: 1$ & 9.74 & 2.04 & 0.06 & 1.21 & -2.44 & 1.48 & 6.04 & 2.23 & 3.21 & 1.32 & -3.75 & 1.63 \\
\hline $1: 3$ & 6.52 & 2.04 & 2.95 & 1.21 & -7.33 & 1.48 & 10.58 & 2.13 & -0.42 & 1.26 & -8.76 & 1.55 \\
\hline $3: 1$ & 6.55 & 1.96 & -1.63 & 1.16 & -7.13 & 1.43 & 2.38 & 2.35 & 1.80 & 1.39 & -1.67 & 1.71 \\
\hline $3: 3$ & 3.13 & 1.96 & -0.74 & 1.16 & -3.14 & 1.43 & 0.27 & 1.95 & 0.33 & 1.16 & -1.34 & 1.43 \\
\hline$M$ & 6.48 & 1.00 & 0.15 & 0.59 & -5.01 & 0.73 & 4.82 & 1.09 & 1.23 & 0.64 & -3.88 & 0.79 \\
\hline
\end{tabular}

the 345 and 567 contexts (reported as a percentage) were $-4.96 \pm 0.85$ and $4.82 \pm 0.91$, respectively. Thus, when the 500 sequence rate was relatively slow, the $500-\mathrm{msec}$ standard IOI was reliably underestimated and when it was relatively fast, it was reliably overestimated.

\section{Comparison of 500-msec Standard IOI Across Test Blocks}

Finally, we considered whether there were changes in relative $\mathrm{CE}$ for the 500-msec standard IOI over the course of the five test blocks. If the observed changes in CE represent a global temporal context effect, then one might expect that distortions in perceived tempo for sequence rates that differ from the global pace should increase over the course of the experiment (as listeners develop a better sense of pace). This result implies that relative CE for the $500 \mathrm{msec}$ standard IOI in the 345 and 567 contexts should be more negative and positive, respectively, in later test blocks than in earlier test blocks. To consider this possibility, we measured CE for the 500-msec standard IOI separately for each test block. Because each participant made a relatively small number of judgments about each comparison IOI in each test block, by-block estimates of CE were obtained by combining data across participants and calculating a single estimate of CE (for each trial block) on the basis of the aggregate data (rather than calculating a separate estimate for each participant). We then used linear regression to examine change in CE across blocks. The results of this analysis are shown in Figure 3, with best fitting regression lines for each context condition. As expected, CE values for $500 \mathrm{msec}$ in the 345 and 567 contexts became progressively more negative and positive, respectively, with increased testing. In general, changes in CE occurred early on in testing (from Block 1 to Block 2) and remained relatively stable until the final test block. The final test block yielded increased distortion for $500 \mathrm{msec}$ in the 567 context, but not in the 345 context.

\section{DISCUSSION}

There are three main findings that were obtained from the preceding experiment. First, numbers of intervals comprising standard and comparison sequences produce

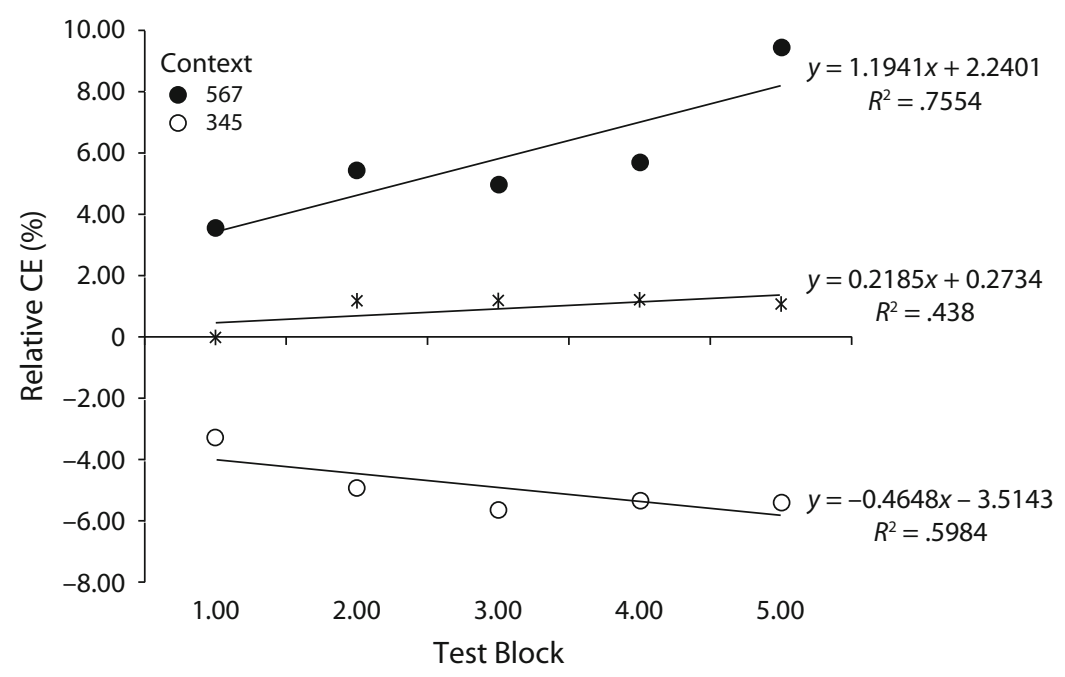

Figure 3. Relative $\mathrm{CE}$ for the 500-msec standard interonset interval as a function of test block (1-5) for the 345 context (open circles) and 567 context (filled circles) with best-fitting regression lines. Included for comparison purposes (stars) is a block analysis of the data for a 456 context condition from McAuley and Miller (2005; Experiment 2). 
independent effects on tempo thresholds (as illustrated by the JND data). Second, standard tempi that are fast in comparison with the global pace are overestimated, whereas standard tempi that were slow in comparison with the global pace are underestimated (as illustrated by the CE data). Third, increasing the number of intervals in the standard sequence produces greater improvements in tempo sensitivity when the standard tempo is different from the global pace than when it is at the global pace. Combined, the results support the view that listeners become attuned to the global pace of their auditory environment, and this can systematically affect listeners' perception of sequence tempo. In the following discussion, we expand on these results and then consider implications of this research for models of timing.

In a previous study, we provided evidence that discrepancies in some studies of tempo sensitivity (Drake \& Botte, 1993; Grondin, 2001; McAuley \& Kidd, 1998) may be explained by three factors: confounding the number of standard and comparison intervals, the use of a fixed versus roving standard IOI, and uncertainty about the number of standard intervals (Miller \& McAuley, 2005). The present investigation extends this research in several important ways.

First, the finding that the standard and comparison sequences make independent contributions to tempo sensitivity provides a valuable replication of Miller and McAuley's (2005) study. As reported previously, increasing either the number of equal intervals in the standard sequence or the number of equal intervals in the comparison sequence reduces tempo thresholds, but the two factors do not appear to interact. The similarity between the studies is highlighted in Figure 4, which shows a comparison of the current JND results with data from the 456-context condition in Miller and McAuley (2005; Experiment 2); all three global temporal contexts show the same general JND pattern. Some physiological support for distinct contributions of the number of standard and comparison intervals to tempo sensitivity comes from a recent ERP study of tempo perception involving the measurement of contingent negative variation, or CNV (Pfeuty, Ragot, \& Pouthas, 2003). In the Pfeuty et al. study, when listeners made relative tempo judgments about standardcomparison pairs of isochronous sequences, increases in CNV amplitude were found during the presentation of the standard sequence, whereas decreases in CNV amplitude were found during the comparison phase. Pfeuty et al. attributed these distinct changes in $\mathrm{CNV}$ to two different tempo discrimination processes (an encoding process and a checking process).

Second, consistent with the view that listeners pick up on the global pace, standard tempi that were relatively fast in comparison with the global pace tended to be overestimated, whereas standard tempi that were relatively slow in comparison with the global pace tended to be underestimated. This finding is highlighted by Figure 5, which compares $\mathrm{CE}$ data from the present study with $\mathrm{CE}$ data from the 456 context in Miller and McAuley (2005; Experiment 2). Simply placing the same 500-msec standard

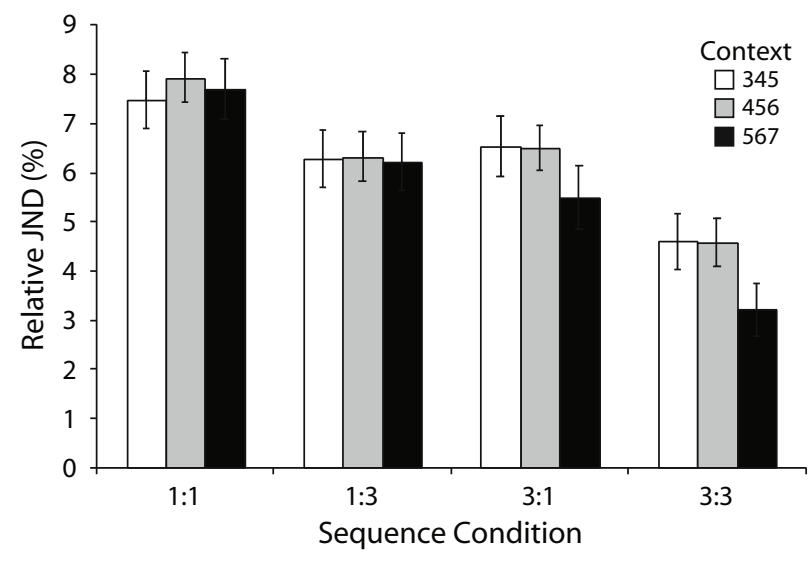

Figure 4. Relative JND as a function of the number of standard and comparison intervals for the 345 and 567 contexts. Included for comparison purposes are data for a 456 context reproduced from McAuley and Miller (2005; Experiment 2).

IOI in different global temporal contexts (so that the standard sequence tempo is relatively fast, relatively slow, or at the global pace) yields distortions in the perceived standard tempo that are in the direction of the global pace.

Third, the reported results support the hypothesis that the number of standard intervals produces the largest effects on tempo sensitivity when standard tempi are different from the global pace. Effects of the number of standard intervals on tempo thresholds were generally larger for the standard tempi that were different from the global pace than for those that were at the global pace. As will be discussed shortly, this finding has important implications for theory, because it suggests that factors that affect JNDs are not independent of factors that affect PSE/CE.

Finally, by examining a broader range of contexts, the present study eliminates the possibility that previously reported conditions yielding null effects of the number of standard intervals on tempo thresholds were due to the specific standard IOIs that were tested (Miller \& McAuley, 2005).

One question that emerges from this work is whether the reported results indeed represent the effects of global temporal context or whether they may be explained by local factors. That is, when the tempo of the standard sequence varies from trial to trial, then the standard tempo on a given trial may potentially carry over to affect estimates of the standard tempo on the subsequent trial. Because the present study did not directly test this factor, we cannot rule out the possibility that local context contributed to our results. However, if the observed context effects were due strictly to local changes in tempo from trial to trial, then we would expect distortions in perceived tempo for sequence rates that differ from the global pace to remain relatively constant over the course of the experiment. This is not what we found. Consistent with the view that a sense of global pace emerges over the course of the experiment, distortions in perceived tempo were larger in later trial blocks than in earlier trial blocks. The observed block effect on CE was somewhat more pronounced in the 


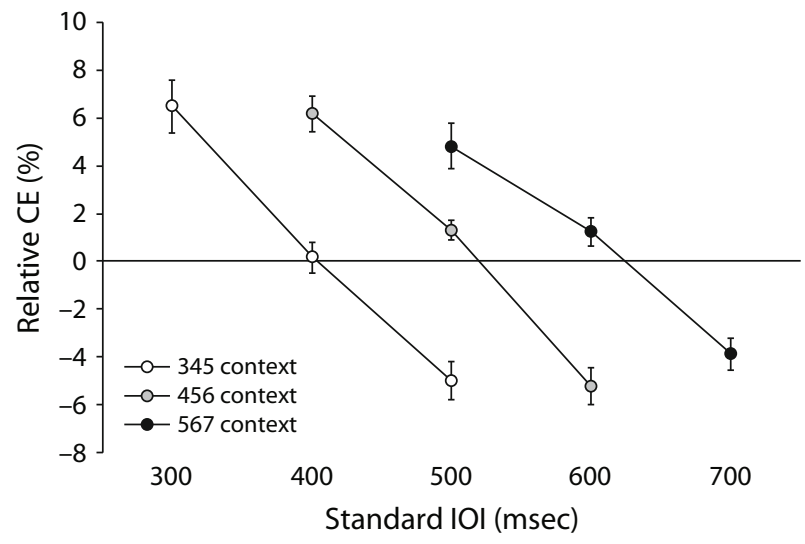

Figure 5. Relative $\mathrm{CE}$ as a function of standard interonset interval (IOI) for the 345 and 567 contexts. Included for comparison purposes are data for a $\mathbf{4 5 6}$ context reproduced from McAuley and Miller (2005; Experiment 2).

567 context than in the 345 context, suggesting that the sense of global pace that emerged may have been overall stronger for participants in the 567 context than those in the 345 context.

Several recent studies report data relevant to the nature of temporal context effects on timing (Jones \& McAuley, 2005; McAuley \& Jones, 2003; Repp, 2001). In Repp (2001), a synchronization-detection paradigm was used to examine participants' abilities to detect and track (via tapping) tempo changes in otherwise isochronous sequences. On some of the trials, no tempo changes occurred within the sequences. Nonetheless, participants frequently reported that they heard tempo changes (i.e., they made false alarms). Notably, the tendency to falsely detect tempo changes increased in magnitude when the standard IOI of the to-be-judged sequence became increasingly different from the average standard IOI (baseline rate) of the set of sequences that participants were exposed to. This finding supports the view that participants' judgments about within-sequence tempo changes are influenced by temporal context, although it is not clear the extent to which this is a local trial-to-trial carry-over effect or a more general effect of global temporal context.

Jones and McAuley (2005) more directly examined the question of local versus global effects of temporal context on perceived sequence timing in a series of experiments that carefully controlled the distributional properties of the time intervals that participants experienced while they were performing a standard-comparison duration judgment task. Three levels of temporal context were identified that appear to affect perceived timing (within-trial context, trial-to-trial carry-over effects, and global temporal context-i.e., global pace). Most relevant for the present investigation, the design of the Jones and McAuley study permitted a systematic examination of trial-to-trial contingencies. Notably, the results showed that global and local (trial-to-trial) temporal context account for distinct sources of variance in time judgment errors. Combined with the present findings, there is increasing evidence that both local and global temporal contexts affect perceived sequence timing.

\section{Implications for Models of Timing}

What are the theoretical implications of these findings? To account for distinct contributions of the number of standard and comparison intervals to tempo thresholds, Miller and McAuley (2005) proposed a generalized multiple-look (GML) model. In the GML model, the original multiple-look model of Drake and Botte (1993) is extended to measure average sampling error for two independent samples, as shown below.

$$
J N D_{n_{1}: n_{2}}=\sqrt{\frac{w\left(J N D_{1: 1}\right)^{2}}{n_{1}}+\frac{(1-w)\left(J N D_{1: 1}\right)^{2}}{n_{2}}} .
$$

The model has two additive terms. The first term refers to the standard sequence, and the second term refers to the comparison sequence, with $n_{1}$ and $n_{2}$ specifying the number of intervals in each sequence, respectively. Consistent with Drake and Botte, tempo JNDs (notated here as $J N D_{n_{1}: n_{2}}$ for isochronous $n_{1}$-interval standard and $n_{2^{-}}$ interval comparison sequences) are based on the observed JND for the single-interval case $\left(J N D_{1: 1}\right)$, and thresholds are similarly predicted to have an inverse relationship with the number of sequence intervals. However, the relative contribution of the standard and comparison sequences to thresholds is permitted to vary using a relative weight parameter, $w .^{2}$

Descriptive application of the GML model is useful for highlighting the main findings in the present study. Specifically, best-fitting estimates of $w$ provide a quantitative interpretation of the relative contribution of the number of standard and comparison intervals to tempo thresholds, with values of $w$ greater than 0.5 indicating a greater contribution of the number of standard intervals to thresholds than the number of comparison intervals. If improvement in tempo sensitivity that is associated with the number of standard sequence intervals is more pronounced for standard IOIs that differ from the mean standard IOI (global pace), then we would expect to find larger $w$ values for those standard IOIs that differ from the global pace than for those equal to the global pace.

Previously, Miller and McAuley (2005) reported that quantitative model fits of the GML model provide a much better account of tempo thresholds than the original multiple-look model. In general, data that are consistent with the original multiple-look model should yield $w$ estimates of approximately 1.0 , but in no instance did they report such an occurrence. In most cases, $w$ estimates were less than 0.5 . This result suggests that the number of comparison intervals plays a larger role in determining tempo thresholds than the number of standard intervals. Moreover, consistent with the view that listeners develop a stable tempo referent for the global pace and that this mediates the relative contribution of the number of standard and comparison intervals to thresholds, larger $w$ estimates were found for the two extreme standard IOIs (400 and $600 \mathrm{msec}$ ) than for the $500-\mathrm{msec}$ standard IOI equal to 
the global pace. The specific estimates of $w$ for the 456 context were $0.50,0.32$, and 0.69 for the $400-, 500-$, and 600 -msec standards, respectively.

Similar — but not identical — patterns of $w$ estimates are obtained for the present study when the GML model is applied to the 345 and 567 contexts. For the 567 context, $w$ estimates are $0.76,0.36$, and 0.76 for the 500-, 600-, and 700-msec standards, respectively; for the 345 context, $w$ estimates are $0.35,0.35$, and 0.5 for the $300-, 400-$, and 500 -msec standards, respectively. A comparison of GML model fits across the two studies permits a few generalizations. First, when all three global contexts are taken into account, estimates of $w$ that are obtained for the global pace are very similar across contexts, ranging from 0.32 to 0.36 . Second, the average value of $w$ decreases as the global pace increases ( 567 context, $M=0.63 ; 456$ context, $M=0.50 ; 345$ context, $M=0.4)$. Third, the pattern of $w$ values across the three standard IOIs comprising each context becomes increasingly asymmetric as the global pace increases; in the 345 context, the asymmetry yielded equal values of $w$ for the 300- and 400-msec standard IOIs.

An important observation to make about the above application of the GML model is that although the GML model provides a descriptive account of tempo threshold data, a weakness of this and other related approaches is that they do not make any explicit predictions about PSE or CE measures. In our view, the GML model provides a nice descriptive summary of the relative contribution of the number of standard and comparison intervals to tempo sensitivity across various global contexts, but it is neutral with respect to the nature of the process.

With respect to the question of timing processes, the GML model is perhaps best accommodated within the theoretical framework of a class of timing models that are typically referred to as interval models (Ivry \& Hazeltine, 1995; Keele et al., 1989; Pashler, 2001). Many of the models developed within this framework are indeed processing models, which posit distinct clock, memory, and decision stages of timing (Church, 2003; Gibbon, Church, $\&$ Meck, 1984). Interval models of this sort typically assume that the clock stage involves a pacemaker, which over time emits a continuous stream of pulses that flow into an accumulator via an attention-controlled switch. The number of pulses that are accumulated during a target time interval, $T$, provides a representation of duration. From the interval perspective, reductions in time sensitivity (increases in thresholds) are typically attributed to increases in the variability of the component clock, memory, or decision processes. In this regard, interval models have been quite successful in modeling thresholds for tasks requiring the discrimination of isolated durations; however, they have been less successful when used to model contextual aspects of timing and associated changes in PSE (see McAuley \& Jones, 2003). Because the findings reported in this article show a dependence between PSE/CE and factors affecting tempo thresholds, the current findings pose a general challenge for interval models of timing.

In the remainder of the discussion, we consider the present findings in light of an alternative view of tim- ing, namely an entrainment (beat-based) perspective. Entrainment models of timing are based on the concept of a self-sustaining (i.e., entrainable) oscillation that peaks in amplitude (a gross measure of neural activity) at regular temporal intervals (Jones \& Boltz, 1989; Large \& Jones, 1999; McAuley, 1995; McAuley \& Jones, 2003; McAuley \& Kidd, 1998). According to this approach, relative time/ tempo judgments are based on the temporal contrast that occurs between the timing of tone onsets in the stimulus sequence (external rhythm) and the periodic timing of expected tone onsets that are generated by the underlying oscillatory timer (hereafter referred to as "beats"). In these models, temporal contrast is typically conceptualized as a relative phase discrepancy. Tone onsets that arrive before an expected tone onset (beat) produce a negative relative phase discrepancy and indicate an increase in tempo (e.g., "faster"); tone onsets that arrive after an expected tone onset (beat) produce a positive relative phase discrepancy and indicate a decrease in tempo (e.g., "slower").

In response to unexpected (early or late) tone onsets, two types of error correction processes operate: period correction and phase correction. Period correction adjusts the period of the internal oscillator (the interbeat interval) so that it eventually matches the IOI of the stimulus sequence. Phase correction ensures that beats are temporally aligned with tone onsets. In order for temporal contrast to provide accurate information about relative tempo, period and phase errors must be minimized. Similar processes have been proposed to operate when individuals are overtly synchronizing with rhythmic sequences by tapping (Mates, 1994; Repp, 2005).

Consider a tempo-discrimination task involving standard-comparison pairs of isochronous sequences. For this task, period and phase correction operate to adjust the interbeat interval so that it comes to match the fixed standard IOI of the standard sequence and so that beats are aligned with tone onsets. In this way, successive interbeat intervals provide an online measure of PSE. The extent of the final temporal match between beats and tone onsets in the standard sequence thus determines both the magnitude of a listener's CEs, as well as the accuracy of information provided by temporal contrasts (phase discrepancies) about the relative tempo of the comparison sequence. When the standard IOI varies from trial to trial, the interbeat interval at the start of the standard sequence is likely to be different from the standard IOI, and several standard intervals may be required before there is a close match between the interbeat interval and the standard IOI. In contrast, when the standard IOI is constant from trial to trial, the interbeat interval established on one trial can effectively carry over to the next trial; thus, less period correction is required to obtain a close match between the interbeat interval and the standard IOI.

Thus, from an entrainment perspective, an effect of the number of standard intervals on tempo thresholds is due to period correction processes, whereas an effect of the number of comparison intervals on tempo thresholds is due to the use of temporal contrast information (i.e., judgments about how aligned successive tone onsets in 
the comparison sequence are with internally generated beats). Thus, consistent with the present findings, increasing the number of standard intervals would be expected to produce improvements in tempo sensitivity in situations that require substantial period correction (e.g., when the standard sequence tempo differs from the global pace) and would not be expected to produce improvements in tempo sensitivity in situations that require little or no period correction (e.g., when the standard sequence tempo is at the global pace - a rate for which the listener may have developed a stable tempo referent). Conversely, increasing the number of comparison intervals is likely to lead to improvements in tempo sensitivity in situations that require little or no period correction (i.e., there is a close match between the interbeat interval and the standard IOI), because it is precisely these situations that the relative phase discrepancies of tone onsets in the comparison sequence (temporal contrasts) provide the most reliable information about the relative tempo of the comparison sequence ("faster" or "slower").

The entrainment account proposed here suggests a slight reinterpretation of the study by Pfeuty et al. (2003) examining electrophysiological correlates of tempo perception. From an entrainment perspective, one possibility is that the increases in CNV amplitude found during the presentation of the standard sequence are attributable to a period-correction process (rather than the operation of a "multiple-look" process), whereas decreases in CNV amplitude found during the presentation of the comparison sequence are associated with the accumulation of temporal contrasts (phase discrepancies). Consistent with this interpretation, Pfeuty et al. proposed that decreases in $\mathrm{CNV}$ amplitude during the comparison phase may be the result of a "checking process" that tests whether the tone onsets of the comparison sequence are at expected times on the basis of the "beats" that are extrapolated from the rhythm of the standard sequence.

In the context of the present findings, one implication of an entrainment interpretation of the Pfeuty et al. (2003) data is that it predicts that the largest increases in CNV amplitude (associated with the presentation of standard sequence) should occur for standard tempi that differ from the global pace, because that is when the most period correction is required. Conversely, an entrainment interpretation predicts that the largest decreases in $\mathrm{CNV}$ amplitude (associated with the comparison phase) should occur for standard tempi that are at the global pace, because that is when the most efficient entrainment occurs, and listeners should be able to most effectively make use of temporal contrast information.

\section{Summary}

Overall, the findings reported in this article support the view that listeners adapt to the global pace of their auditory environment, which has an impact on local judgments about sequence tempo. Specifically, in the context of a tempo-discrimination paradigm involving standardcomparison pairs of isochronous tone sequences, support was found for the hypothesis that increasing the number of standard sequence intervals produces greater improve- ments in tempo sensitivity when the tempo of the standard sequence is different from the global pace than when it is at the global pace. Moreover, consistent with earlier work, standard and comparison sequences make independent contributions to tempo thresholds. An entrainment account of these findings leads to testable predictions about electrophysiological correlates of tempo perception.

\section{AUTHOR NOTE}

A portion of this research was supported by PHS Grant AG20560. The authors thank Bruno Repp and Rolf Ulrich for their many helpful comments and constructive suggestions for improvements regarding earlier versions of the manuscript. The authors also thank the members of the Timing Research Group at Bowling Green State University for their assistance during the completion of this project. Correspondence concerning this article should be addressed to J. D. McAuley, Department of Psychology, Bowling Green State University, Bowling Green, OH 43403 (e-mail: mcauley@bgnet.bgsu.edu).

\section{REFERENCES}

Aвel, S. M. (1972). Discrimination of temporal gaps. Journal of the Acoustical Society of America, 52, 519-524.

Allan, L. G. (1979). The perception of time. Perception \& Psychophysics, 26, 340-354.

Church, R. M. (2003). A concise introduction to scalar timing theory. In W. H. Meck (Ed.), Functional and neural mechanisms of interval timing (pp. 3-22). Boca Raton, FL: CRC Press.

Creelman, C. D. (1962). Human discrimination of auditory duration. Journal of the Acoustical Society of America, 34, 582-593.

Drake, C., \& Botte, M.-C. (1993). Tempo sensitivity in auditory sequences: Evidence for a multiple-look model. Perception \& Psychophysics, 54, 277-286.

FraIsse, P. (1963). The psychology of time. New York: Harper \& Row.

Friberg, A., \& Sundberg, J. (1995). Time discrimination in a monotonic, isochronous sequence. Journal of the Acoustical Society of America, 98, 2524-2531.

GetTy, D. J. (1975). Discrimination of short temporal intervals: A comparison of two models. Perception \& Psychophysics, 18, 1-8.

Gibbon, J., Church, R. M., \& Meck, W. H. (1984). Scalar timing in memory. Annals of the New York Academy of Sciences, 423, 52-77.

Grondin, S. (2001). Discriminating time intervals presented in sequences marked by visual signals. Perception \& Psychophysics, 63, 1214-1228.

Ivry, R. B., \& Hazeltine, R. E. (1995). Perception and production of temporal intervals across a range of durations: Evidence for a common timing mechanism. Journal of Experimental Psychology: Human Perception \& Performance, 21, 3-18.

JonES, M. R. (1976). Time, our lost dimension: Toward a new theory of perception, attention, and memory. Psychological Review, 83, 323-355.

Jones, M. R., \& Boltz, M. (1989). Dynamic attending and responses to time. Psychological Review, 96, 459-491.

Jones, M. R., \& McAuley, J. D. (2005). Time judgments in global temporal contexts. Perception \& Psychophysics, 67, 398-417.

Jones, M. R., \& Yee, W. (1997). Sensitivity to time change: The role of context and skill. Journal of Experimental Psychology: Human Perception \& Performance, 23, 693-709.

Keele, S. W., Nicoletti, R., Ivry, R. I., \& Pokorny, R. A. (1989). Mechanisms of perceptual timing: Beat-based or interval-based judgements? Psychological Research, 50, 251-256.

Large, E. W., \& Jones, M. R. (1999). The dynamics of attending: How people track time-varying events. Psychological Review, 106, 119-159.

LASHLEY, K. S. (1951). The problem of serial order in behavior. In L. A. Jeffress (Ed.), Cerebral mechanisms in behavior: The Hixon Symposium (pp. 112-146). New York: Wiley.

Macmillan, N. A., \& Creelman, C. D. (1991). Detection theory: A user's guide. Cambridge: Cambridge University Press.

MATES, J. (1999). A model of synchronization of motor acts to a stimulus sequence: I. Timing and error corrections. Biological Cybernetics, 70, 463-473. 
McAuley, J. D. (1995). Perception of time phase: Toward an adaptive oscillator model of rhythmic pattern processing. Unpublished dissertation, Indiana University, Bloomington.

McAuley, J. D., \& Jones, M. R. (2003). Modeling effects of rhythmic context on perceived duration: A comparison of interval and entrainment approaches to short-interval timing. Journal of Experimental Psychology: Human Perception \& Performance, 29, 1102-1125.

MCAuley, J. D., \& KIDD, G. R. (1998). Effect of deviations from temporal expectations on tempo-discrimination of isochronous tone sequences. Journal of Experimental Psychology: Human Perception \& Performance, 24, 1786-1800.

Michon, J. A. (1964). Studies on subjective duration: I. Differential sensitivity in the perception of repeated temporal intervals. Acta Psychologica, 22, 441-450.

Miller, N. S., \& MCAuley, J. D. (2005). Tempo sensitivity in isochronous tone sequences: The multiple-look model revisited. Perception \& Psychophysics, 67, 1150-1160.

Pashler, H. (2001). Perception and production of brief durations: Beatbased versus interval-based timing. Journal of Experimental Psychology: Human Perception \& Performance, 27, 485-493.

Pfeuty, M., Ragot, R., \& Pouthas, V. (2003). Processes involved in tempo perception: A CNV analysis. Psychophysiology, 40, 69-76.

Repp, B. H. (2001). Processes underlying adaptation to tempo changes in sensorimotor synchronization. Human Movement Science, 20, 277-312.

REPP, B. H. (2005). Sensorimotor synchronization: A review of the tapping literature. Psychonomic Bulletin \& Review, 12, 969-992.

Rousseau, L., \& Rousseau, R. (1996). Stop-reaction time and the internal clock. Perception \& Psychophysics, 58, 434-448.

Schulze, H. H. (1989). The perception of temporal deviations in isochronic patterns. Perception \& Psychophysics, 45, 291-296.

Small, A. M., \& Campbell, R. A. (1962). Temporal differential sensitivity for auditory stimuli. American Journal of Psychology, 75, 401-410.

ten Hoopen, G., \& AKerboom, S. (1983). The subjective tempo dif- ference between interaural and monaural sequences as a function of sequence length. Perception \& Psychophysics, 34, 465-469.

TodD, R. E., Boltz, M., \& Jones, M. R. (1989). The Midilab auditory research system. Psychomusicology, 8, 83-96.

VIERORDT, K. (1868). Der Zeitsinn nach Versuchen. Tübingen: Laupp.

Woodrow, H. (1951). Time perception. In S. S. Stevens (Ed.), Handbook of experimental psychology (pp. 1224-1236). New York: Wiley.

\section{NOTES}

1. For this task, there is typically a "gap" between the standard and comparison sequences that is equal to twice the standard IOI. The purpose of the gap is to distinguish the standard and comparison sequences for the listeners. The effect of the gap on tempo discrimination performance has been investigated previously by a number of researchers (e.g., Grondin, 2001; McAuley \& Jones, 2003).

2. A close inspection of Equation 2 shows that in the GML model, thresholds decrease with increases in $n_{1}$ and $n_{2}$, with the parameter $w$ modulating the contribution of each sequence in a manner similar to a weighted average. When $w=1$, the predicted JND in tempo- $J N D_{n_{1}: n_{2}}$-is determined by the number of standard intervals only and the model reduces to the original multiple-look model that is depicted in Equation 1. In contrast, when $w=0$, thresholds are determined by the number of comparison intervals only, a possibility that was not explicitly considered by Drake and Botte (1993). Finally, when $w$ takes on intermediate values, thresholds are influenced by a weighted combination of the number of intervals in both sequences. Thus, the relative contribution of the number of standard and comparison intervals to tempo thresholds varies with $w$. Values of $w$ greater than 0.5 indicate that thresholds are determined more by the number of standard intervals than by the number of comparison intervals, whereas values less than 0.5 indicate the opposite.

(Manuscript received November 23, 2005; revision accepted for publication November 13,2006.) 